Polymer

April 2018, Volume 142, Pages 209-217

http://dx.doi.org/10.1016/i.polymer.2018.03.034

http://archimer.ifremer.fr/doc/00433/54467/

(c) 2018 Published by Elsevier Ltd.

\title{
Strain-induced crystallization in an unfilled polychloroprene rubber: Kinetics and mechanical cycling
}

\author{
Le Gac Pierre-Yves ${ }^{1,}{ }^{*}$, Albouy Pierre-Antoine ${ }^{2}$, Petermann Denis ${ }^{2}$ \\ 1 IFREMER ,Centre de Bretagne, Marine Structures Laboratory, BP70, 29280, Plouzané, France \\ ${ }^{2}$ Laboratoire de Physique des Solides, CNRS, Université Paris-Sud, Université Paris-Saclay, 91405, \\ Orsay, France \\ * Corresponding author : Pierre-Yves Le Gac, email address : pierre.yves.le.gac@ifremer.fr
}

\begin{abstract}
:
Polychloroprene rubber is often quoted as an example of strain-crystallizing elastomer; however, this fundamental property, known to impart good mechanical properties and fatigue resistance, has received much less attention in this class of compounds than natural rubber. The present paper relates systematic investigations combining mechanical and X-ray diffraction measurements. Crystallization kinetics are first investigated based on tensile impact tests. An approximately logarithmic timedependence is found for the crystalline content and the time-constants are estimated at various temperatures. The impact of strain-induced crystallization on stress-strain curves under mechanical cycling conditions is further assessed. It is demonstrated that one main effect of strain-induced crystallization is a partial relaxation of the strain experienced by the amorphous fraction, although stress-hardening is simultaneously observed. Based on the stress-optical law, the contribution of the amorphous phase to the stress is shown to level off after crystallization onset, and stress-hardening is attributed to crystallite networking. The crystallite network rapidly disintegrates at the beginning of recovery, and the stress-optical law shows that the amorphous fraction becomes the only contributor to the stress at still non-negligible crystalline contents.
\end{abstract}




\section{Graphical abstract}

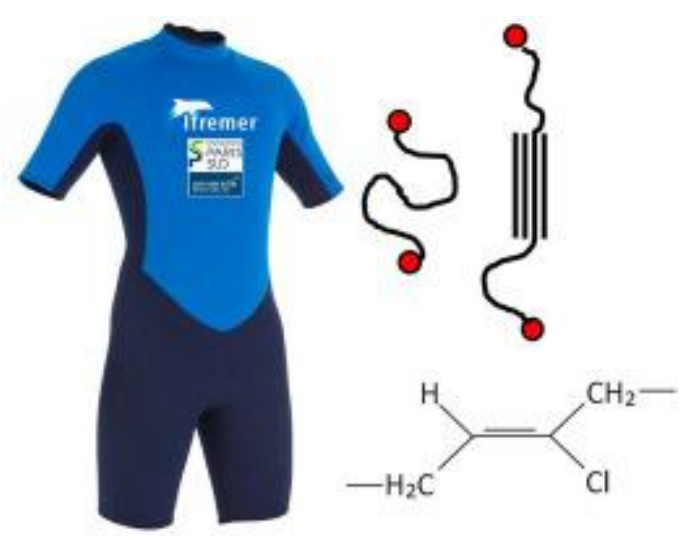

\section{Highlights}

Temperature effect on SIC in polychloroprene rubber is investigated. logarithmic time-dependence for all tested temperatures. Impact of strain on melting temperature is well characterized. Stress hardening during tensile test is due to crystallite networks whereas the amorphous phase only contributes to the stress when no crystallites are created.

Keywords : Polychloroprene, Strain-induced crystallization, X-ray 


\section{Introduction}

Chloroprene rubbers (CR) are a class of specialty elastomers widely used in many demanding industrial applications like coatings, sealing, or as part of structures in a marine environment [1]. Their better durability and lower sensitivity to swelling by nonpolar solvents and lower oxidation rate compared for instance to natural rubber (NR) is due to the presence of a chlorine atom close to the double bond, that reduces its reactivity [2,3]. CRs belong to the class of strain-crystallizing elastomers, and strain-induced crystallization (SIC) is known to increase dramatically both the fracture properties and fatigue life [4, 5]. It has received much less attention in CRs compared to NRs, and many of the studies date back to the 60's when the crystallization process was highlighted in this class of compounds. Huge progress made in the production and detection of X-rays has led to a recent renewal of SIC studies coupling mechanical and diffraction measurements, but this effort essentially benefited NRbased compounds [6]; we only know of one such study devoted to CR [7]. The need for a better knowledge of the impact of SIC in a generic unfilled W-type CR prompted the present work. Elastomers are most often used in dynamic conditions and the efficiency of SIC as a reinforcement mechanism is thus intimately related to its kinetics. The first section of this paper focuses on this point. The impact of SIC during mechanical cycling is then examined.

There is a large literature on SIC in elastomers and we will briefly recall what distinguishes this phenomenon from quiescent crystallization (QC) (i.e. thermally-induced crystallization at zero strain; the term "cold crystallization" is also found in the literature). Although SIC is common to a large number of elastomers, none has received as much attention as NR, which appears as the main example for strain-crystallizing elastomers, and quite often as the only reference. A first important point is that crystal structures are in general similar for QC and SIC [8-9]. QC in elastomers that readily strain-crystallize is quite often a slow process even at optimal degrees of super-cooling, and crystallization half-times are counted in hours or days [10-11]; folded conformation is most probable and spherulitic or lamellar growth is observed under some conditions [6-8]. The crystallization kinetics progressively accelerate with increasing applied elongation and crystallites become oriented with the chain-axis on average parallel to the stretching direction [11, 15-17]. The formation of extended crystallites becomes most probable at elongations where the kinetics become rapid (sub-second times): this can be defined as the effective SIC domain. Shish-kebab formation is possible in the intermediate range between QC and SIC [17]. This transition from folded to extended conformation is also reproduced by Monte-Carlo simulations [18]. The melting temperature $T_{M}(\lambda)$ increases rapidly with elongation $\lambda$ in the SIC range and may exceed the incipient melting temperature by more than $100^{\circ} \mathrm{C}$ [19]. This effect is due to the combination of two factors that constitute the ingredients of the theory developed by Flory [20]: there is first a reduction of the entropy cost for amorphous chain fragments that enter into a crystallite as they are pre-extended: a similar effect governs flow-induced crystallization during injection of polymers melts. The second entropic mechanism is specific to elastomers: a chain fragment embedded in a crystallite adopts a fully extended conformation; as both ends of the chain are constrained by cross-links, the chain section 
outside the crystallite is partially relaxed. Although an improvement of the mechanical properties by SIC is well-established, the actual process at its origin is still debated: the fact that crystallites act as physical cross-links or fillers is usually evoked. We recently proposed that both the partial relaxation of the molten fraction by SIC and crystallite networking were playing a major role [21]. These points will be discussed.

A first section contains experimental details related to the material formulation, diffraction apparatus and data treatment. The results obtained are presented in the following section. It begins with a short paragraph on the effect of elongation upon melting temperature. The kinetics of the crystallization that follows an ultra-rapid extension are then analyzed for various final elongations and temperatures. Finally the impact of crystallization on mechanical cycling is assessed for different test temperatures.

\section{Experimental section}

\subsection{Materials}

The polychloroprene gum used for the present material is a homopolymer (W-type) whose exact microstructure has been assesed by NMR: trans-1,4 (91.8\%), cis-1,4 (6\%), 1,2units $(1.5 \%)$ and 3,4-units $(0.7 \%)$. The chemical disorder decreases the melting temperature and ability to QC compared to the stereoregular polymer, which allows application as a rubber [16]. The gum is cross-linked by sulfur vulcanization carried out at $160^{\circ} \mathrm{C}$ for 10 minutes in a custom-made compression mold (see detailed formulation in Table 1). Samples have a dumbbell form $20 \mathrm{~mm}$ in length and $7 \mathrm{~mm}$ in width. The sample thickness is critical as chlorine is an effective absorbing element at the X-ray wavelength presently used $(0.1542 \mathrm{~nm})$. The optimal thickness for diffraction at this photon energy $(8 \mathrm{keV})$ is ca. $0.3 \mathrm{~mm}$; sample thickness was fixed at ca. $0.5 \mathrm{~mm}$ so that the optimal value is attained at typically half the maximum applied elongation. Samples were systematically extended a few times before use to suppress the Mullins effect; they were then heated to $60^{\circ} \mathrm{C}$ to eliminate any remaining crystalline phase. The estimation of the cross-link density $v$ is based on mechanical measurements using the Moonley-Rivlin formula for the engineering stress $2\left(C_{1}+C_{2} / \lambda\right)\left(\lambda-1 / \lambda^{2}\right)$ (elongation: $\lambda$ ). It is assumed that $v=2 C_{1} / R T$ ( $R$ gas constant, $T$ absolute temperature; see Table1) [23].

\begin{tabular}{|c|c|}
\hline CR (W type) & $100 \mathrm{phr}$ \\
\hline $\mathrm{MgO}$ & $4 \mathrm{phr}$ \\
\hline $\mathrm{ZnO}$ & $5 \mathrm{phr}$ \\
\hline Sulfur & $1.5 \mathrm{phr}$ \\
\hline Stearic Acid & $0.5 \mathrm{phr}$ \\
\hline $\mathrm{HPPD}$ & $3 \mathrm{phr}$ \\
\hline Crosslink Density $\left(10^{4} \mathrm{~mol} / \mathrm{cm}^{3}\right)$ & $0.746(186 \mathrm{monomers} / \mathrm{chain})$ \\
\hline $\mathrm{C}_{2} / \mathrm{C}_{1}$ & 2 \\
\hline
\end{tabular}

Table 1: Detailed formulation for the polychloroprene rubber used in this study 


\subsection{Stretching set-up}

Although it is commonly asserted that time-resolved X-ray diffraction on elastomers requires the high flux delivered by synchrotron facilities, we have previously shown that usable data may be collected with a simple laboratory set-up [21, 24]. The apparatus is basically a symmetric stretching machine mounted on a rotating anode generator; the $\mathrm{K}_{\alpha}$ radiation emitted by the copper anode is selected by a doubly-curved graphite monochromator that provides high flux (ca. $510^{9} \mathrm{ph} / \mathrm{s}$ when operated at $40 \mathrm{kV}, 40 \mathrm{~mA}$ ). The drawing speed for mechanical cycling can be selected in the range $1-800 \mathrm{~mm} / \mathrm{min}$ for temperatures between $0^{\circ} \mathrm{C}$ and $100^{\circ} \mathrm{C}$. The set-up is normally equipped with an indirect illumination CCD X-ray camera whose main disadvantage is a long transfer time (around 6s); it is well-adapted to mechanical cycling at moderate strain-rate, but a strategy of cumulative acquisition becomes mandatory for kinetics measurements: such a stroboscopic technique has been used for the evaluation of the crystallization rate and induction period in NR samples by different authors [23, 25-26]. It can be now replaced by a hybrid pixel detector that combines a better efficiency (ca.98\% at $8 \mathrm{keV}$ ) and shorter transfer time (ca. $0.25 \mathrm{~s}$ ) when more rapid data acquisition is needed [21]. It makes an evaluation of crystallization kinetics by the tensile impact test method possible to some extent in the laboratory, while it has been up to now essentially confined to synchrotron facilities; see for instance [28] for a pioneering work on polyisobutylene and [29-31] for more recent studies on NR. The new high-speed extension system consists of two pneumatic springs fixed at the clamp positions; compressed air at 6 bars stored in a buffer bottle is admitted into the springs by the opening of a large diameter solenoid valve. It simultaneously triggers a sequence of acquisition for the X-ray camera and the load cell. The step duration has been evaluated to be around 10ms using optical sensors. For measurements where a sensitive load cell is necessary, the sample pull can be ensured by a single spring while the load cell remains at a fixed opposite position and experiences no shock. We presently prefer using two opposing pneumatic springs to achieve symmetric stretching.

\subsection{Data processing}

A representative X-ray diffraction pattern of a CR sample partially crystallized by SIC is presented in Figure 1. The blind vertical line (1) corresponds to the gap between the two detecting elements of the detector; the central circular white spot is the shadow of the beamstop. The "halo" (2) is due to scattering by the amorphous fraction and mainly originates from inter-chain positional correlations [32]. Diffraction features (3) and (4) come from the crystallized fraction. The unit cell of polychloroprene is orthorhombic with four polymer chains passing through and parallel to the c-axis [8]; this axis gets oriented along the stretching direction and the reflections with constant $l$-index are located on the black dotted lines. The high-degree of orientation of the c-axis is shown by the restricted angular spread of the intense equatorial spot (3) that combines (120) and (200) reflections (14.06 nm ${ }^{-1}$ ) [8, 33]. Due to the above mentioned in-chain substitutional disorder, reflections with indices $l= \pm 1$ are blurred into a diffuse streak $\left(13.37 \mathrm{~nm}^{-1}\right)(4)$; as the disorder is located along the c-axis, it affects equatorial reflections with zero $l$-index. The three homogeneous lines (5) arise from the added zinc oxide (see Table 1). 


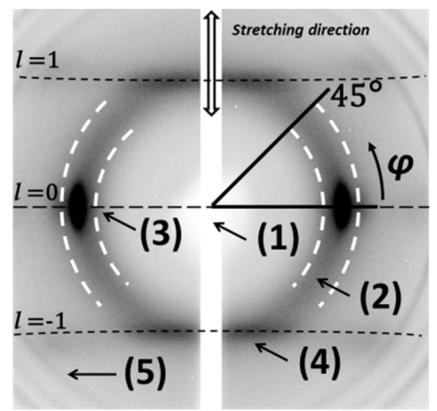

Figure 1. Representative diffraction pattern of a partially strain-crystallized CR sample (elongation: 5, room temperature, pixel-type detector, exposure time: $3 \mathrm{~s}$ ).

The two parameters most relevant for the present study and that can be extracted from the diffraction patterns are a crystallinity index and the anisotropy coefficient for the amorphous halo. An absolute determination of the degree of crystallinity in a semi-crystalline polymer by X-ray diffraction requires in principle an evaluation of the intensity scattered by both the crystalline and amorphous fractions in the whole space [34]. Under practical conditions, measurements are restricted to a limited zone where the most significant part of the scattering gets concentrated. In this way one defines a crystallinity index (CI) as the ratio of the area under the crystallization peaks to the total scan area; if the zone is correctly chosen, $\mathrm{CI}$ is expected to be close to the degree of crystallinity. We presently use the zone encompassed between the white dotted lines and limited to $\pm 45^{\circ}$ to avoid interference with the diffuse streak (4) (see Figure 1). The angular $\varphi$-scan is composed of a peak associated to the $(120)+(200)$ reflections superimposed on a background that combines amorphous, air and Compton scatterings. The removal of the air scattering contribution is described elsewhere [24]; the Compton contribution is low as regards to the scattering angles but has nonetheless been evaluated and substracted [35]. The crystalline peak is adjusted by a Pearson VII function, which is basically a lorentzian function raised to a power $\mu$; this type of function is often used in crystallography due to its versatility [36]. The $\mu$ exponent is fixed at 2 based on test measurements in highly extended and crystallized samples. The unixial extension induces a modulation of the amorphous intensity that can be expanded in even powers of $\cos \varphi$ for symmetry reasons; the development can be stopped at $A+B \cos ^{2} \varphi$ for the whole elongation range. An "order parameter" $\left\langle P_{2}^{R X}\right\rangle=\frac{1}{2}\left(3\left\langle\cos ^{2} \varphi\right\rangle-1\right)$ may be computed from $A$ and $B$, that proved very sensitive to the strain-state for NR-based materials [21, 37]; this sensitivity is presently significantly diminished by the limited amplitude of the angular scan. Mitchell et al. have shown that by making reasonable assumptions, $\left\langle P_{2}^{R X}\right\rangle$ can be assumed proportional to the Hermans coefficient $\left\langle P_{2}\right\rangle$ that quantifies the segmental orientation within the only amorphous phase [38-39]. This is an advantage compared to evaluations of $\left\langle P_{2}\right\rangle$ by infra-red dichroism or birefringence where contributions of the amorphous and crystallized phases are difficult to separate [40-41]. The knowledge of $\left\langle P_{2}^{R X}\right\rangle$ over the whole elongation-range offers thus a unique opportunity to access the strain-state of the amorphous fraction after SIC onset and to evaluate its contribution to stress. This procedure was recently applied to the case of NR $[21,37]$. 


\section{Results and Discussion}

\subsection{Melting temperature and elongation}

The assesment of the relationship between elongation and melting temperature in NR or CR has been one test for Flory's theory [15, 19, 42]; the agreement is found to be semiquantitative at best. The question is still debated and remains the object of publications $[43,44]$. Our purpose is actually quite different and restricted to the evaluation of the minimal elongation at which the crystalline phase is still present at a given temperature; such a knowledge is a prerequisite to any discussion of the SIC effect during mechanical cycling. A measurement of the melting temperature in the quiescent state is also included for comparison; in that case, samples are left to crystallize for one week in a refrigerator at $-20^{\circ} \mathrm{C}$ prior to transfer to the X-ray set-up. Stretched samples crystallize more readily and they were maintained at around $-9^{\circ} \mathrm{C}$ for a few hours in the setup until the crystalline content was sufficient to permit reliable measurements by X-ray diffraction; they were then warmed up step-wise until complete melting. Breakage occurred systematically before melting for the higher elongations investigated, and the melting temperature was obtained by linear extrapolation to zero for the CI. Data presented here are averages on two samples.

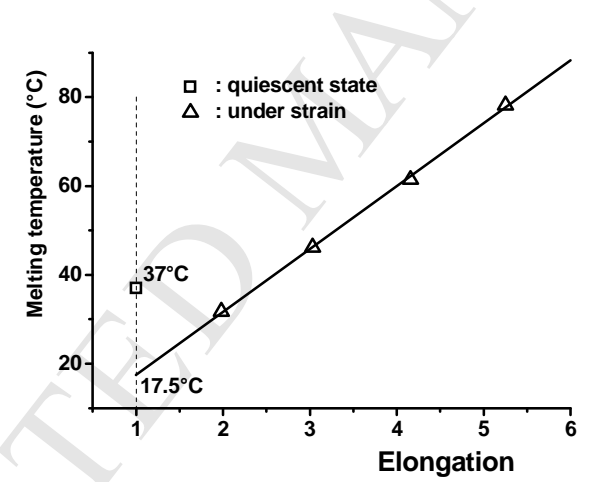

Figure 2. Effect of elongation on the melting temperature.

A linear dependence of the SIC-melting temperature on elongation is observed, $T_{M}(\lambda) \approx 3.4+14.1 \lambda\left({ }^{\circ} \mathrm{C}\right.$ ) (Eq.1) (see Figure 2). The extrapolated temperature at $\lambda=1$ is around $17.5^{\circ} \mathrm{C}$, significantly lower than the melting temperature for samples crystallized at $20^{\circ} \mathrm{C}$ in the quiescent state $\left(37^{\circ} \mathrm{C}\right)$; this important result is discussed in the "mechanical cycling" section. A similarly linear relation is found for NR, for which the slope is in the range 25-30 independent of the cross-link density [19, 42]. It should be noted that an approximately linear behavior is actually predicted in this elongation range by Flory's theory, but the slope is expected to depend upon the cross-link density. The ability to straincrystallize appears higher for NR and the absence of chemical disorder could be a first explanation. The lower melting enthalpy in NR $\left(4.39 \mathrm{kJmol}^{-1}\right)$ compared to $\mathrm{CR}\left(8.37 \mathrm{kJmol}^{-1}\right)$ can also be invoked [20]. 


\subsection{Crystallization kinetics}

Tensile impact tests have been conducted at different step-end elongations and temperatures; the only limitation is sample breakage and we opted for maximal values of respectively 5.3 and $40^{\circ} \mathrm{C}$. Extension was started from the zero-elongation state although the only requisite for the initial strain state is that no crystallization develops during the time necessary to start the experiment. Each acquisition set consists of three successive sequences of 30 diffraction frames each with exposure durations $0.3 \mathrm{~s}, 3 \mathrm{~s}$ and $30 \mathrm{~s}$. The data transfer time is ca. $0.3 \mathrm{~s}$ and it must be correctly included in the calculation of the elapsed crystallization time.
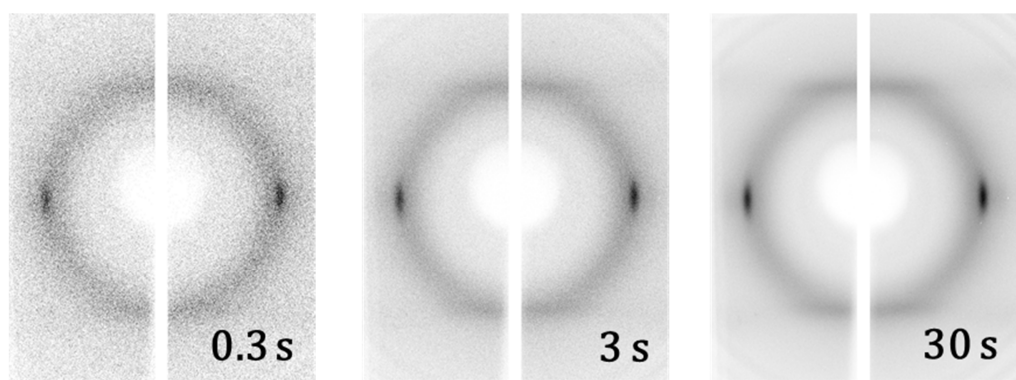

Figure 3. Representative diffraction patterns collected during a tensile test sequence with different acquisition durations (final elongation: 4.8 ; temperature: $20^{\circ} \mathrm{C}$ )

Diffraction patterns collected during an acquisition sequence are shown in Figure 3 at the successive exposure times; such measurements have been systematically carried out on a minimum of four samples for each set of temperatures and step amplitudes to increase the representativeness of the results. An illustration of the data dispersion related to the sample variability is given in Figure 4-A and B; the observed differences are most probably due to slight variations in the sample shape that may affect the exact elongation value at the end of the step. The counting statistic remains correct even at the shorter exposure times for CI; this is not the case for $\left\langle P_{2}^{R X}\right\rangle$, mainly as a consequence of the limited angular range of the intensity scan. The best that can be ascertained is a decrease of $\left\langle P_{2}^{R X}\right\rangle$ with elapsed time that illustrates the relaxing effect of SIC. The horizontal dotted line (Figure 4-B) indicates the initial $\left\langle P_{2}^{R X}\right\rangle$ value at zero-time based on equation (2).

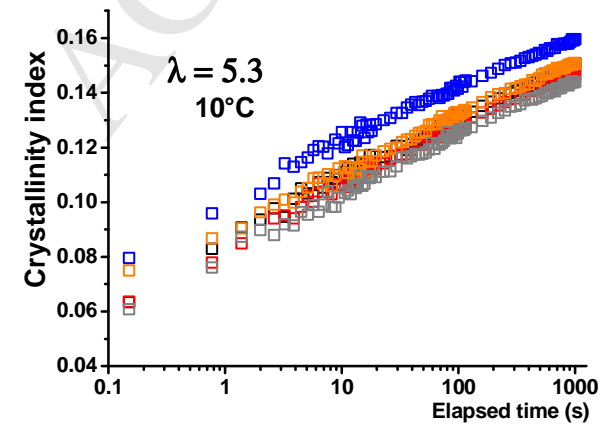

$-\mathrm{A}-$

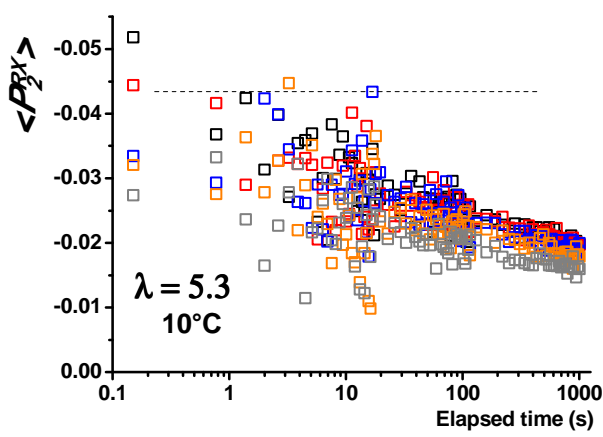

$-\mathrm{B}-$ 
Figure 4. Time dependence of the crystallinity index (left) and orientation parameter $\left\langle P_{2}^{R X}\right\rangle$ (right) upon crystallization time for four samples of the same batch.

The averaged values of $\mathrm{CI}$ at the different elongations and test temperatures are collected in Figure 5; the horizontal error bars correspond to the acquisition time for each frame. A logarithmic time-dependence appears to be obeyed, except for the lower investigated elongation where significant deviations are observed at shorter times. The kinetic law is thus written: $C I(t) \approx A \cdot \log \left(t / \tau_{0}\right)$ (eq.3), where $t$ is the elapsed time and $\tau_{0}$ is tentatively interpreted as an induction time necessary for crystallization to develop after step completion. Concerning this last point, some reservations need to be noted: the step duration is about $10 \mathrm{~ms}$ which per se introduces an uncertainty on the time origin. Induction times $\tau_{0}$ shorter than $10 \mathrm{~ms}$ can thus be questioned.
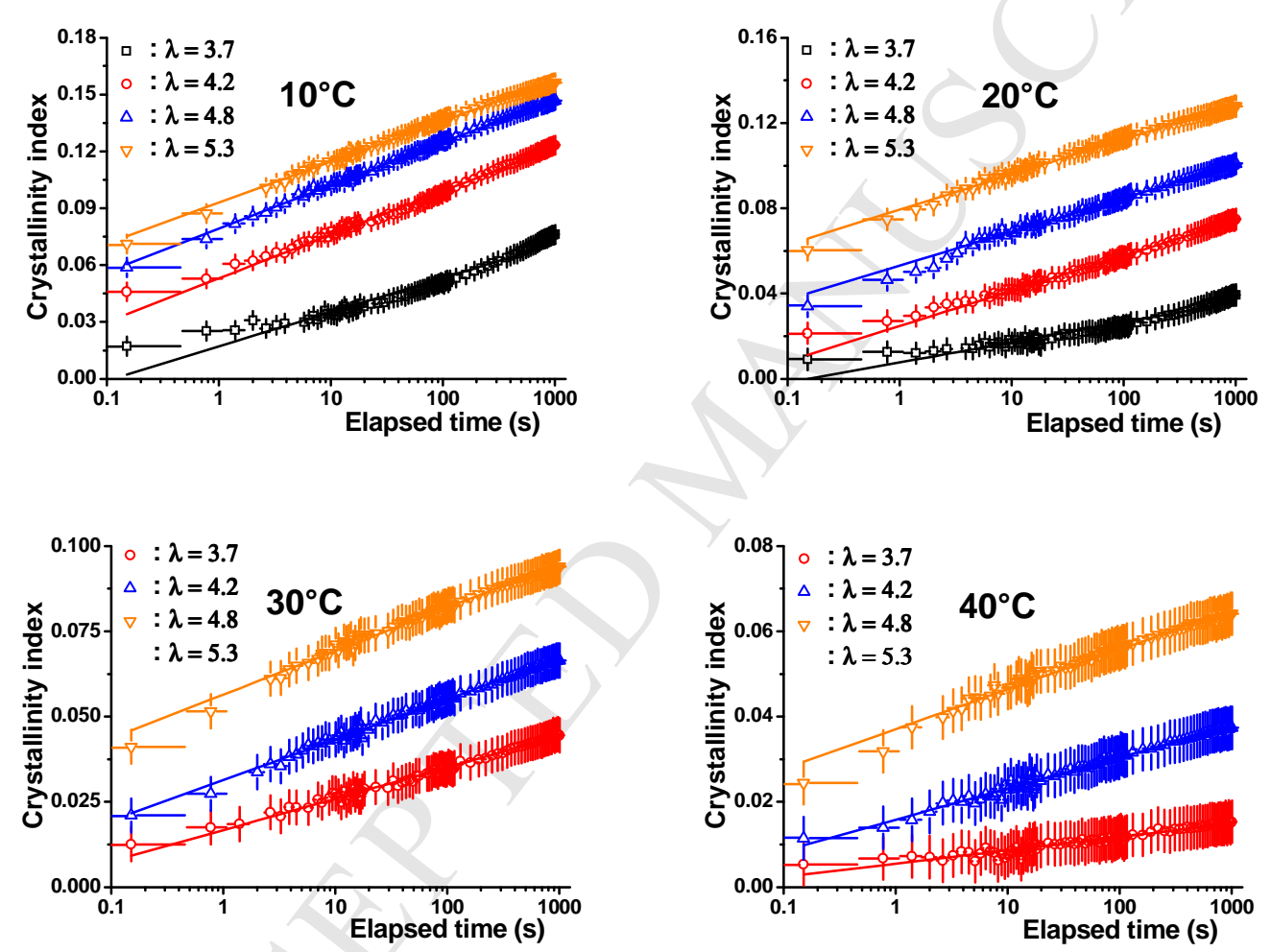

Figure 5. Time-dependence of the averaged crystallinity index at different elongations and temperatures.

\begin{tabular}{|c|c|c|c|}
\hline Elongation & Temperature & $\mathrm{A}$ & $\tau_{0}(\mathrm{~ms})$ \\
\hline 3.7 & $10^{\circ} \mathrm{C}$ & 0.018 & 110 \\
\hline 3.7 & $20^{\circ} \mathrm{C}$ & 0.0094 & 152 \\
\hline 4.2 & $10^{\circ} \mathrm{C}$ & 0.023 & 5 \\
\hline 4.2 & $20^{\circ} \mathrm{C}$ & 0.0165 & 31 \\
\hline 4.2 & $30^{\circ} \mathrm{C}$ & 0.0092 & 15 \\
\hline 4.2 & $40^{\circ} \mathrm{C}$ & 0.0031 & 18 \\
\hline 4.8 & $10^{\circ} \mathrm{C}$ & 0.0228 & 0.3 \\
\hline 4.8 & $20^{\circ} \mathrm{C}$ & 0.0159 & 0.5 \\
\hline 4.8 & $30^{\circ} \mathrm{C}$ & 0.0119 & 2 \\
\hline 4.8 & $40^{\circ} \mathrm{C}$ & 0.0073 & 7 \\
\hline 5.3 & $10^{\circ} \mathrm{C}$ & 0.0215 & 0.05 \\
\hline 5.3 & $20^{\circ} \mathrm{C}$ & 0.0165 & 0.02 \\
\hline 5.3 & $30^{\circ} \mathrm{C}$ & 0.0128 & 0.04 \\
\hline 5.3 & $40^{\circ} \mathrm{C}$ & 0.0093 & 0.1 \\
\hline
\end{tabular}


Table 2. Values for parameters $A$ and $\tau_{0}$ input to equation (3).

Values found for parameters $A$ and $\tau_{0}$ are collected in Table 2. As noted above, very short induction times must be taken with caution due to the transient step duration. Whatever restrictions can be made, as-found $\tau_{0}$ values are undoubtedly indicative of a tendency and it can be concluded that SIC induction in CR is a rapid phenomenon, even at the lower extensions. It first appears that $\tau_{0}$ mostly depends upon elongation and only weakly upon temperature. One may define a temperature-averaged induction period that is plotted in Figure 6 -A; it can be adjusted by exponential elongation-dependence $\left\langle\tau_{0}\right\rangle=1.310^{11} \exp (-\lambda / 0.19)$ (eq.4). The slope $A$ displays an opposite behavior and mostly depends upon temperature and not elongation, except perhaps for the lower elongation. The temperature dependence of the elongation-averaged slope is plotted in Figure 6-B and closely follows a linear dependence $\langle A\rangle=0.02725-5.2410^{-4} \vartheta\left({ }^{\circ} C\right)$ (eq.5). Equations 4 and 5 are not expected to give more than a rough estimate of the crystallization kinetics for a chosen set of temperature and elongation.

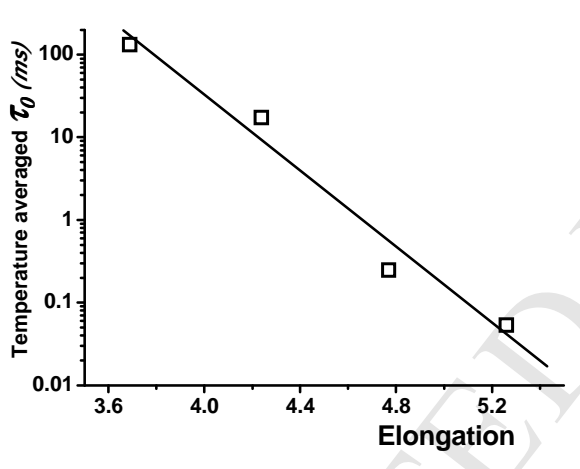

A-

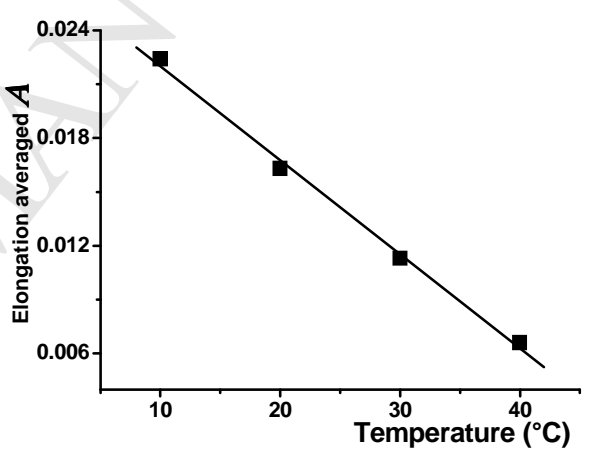

$-\mathrm{B}-$

Figure 6. A- Temperature-averaged induction time versus elongation. B- Elongationaveraged slope versus temperature.

The origin of the observed time-dependence is now discussed. It must first be stressed that many different kinetic laws may approach a logarithmic form; it was chosen for convenience, as a way to define a minimum number of significant parameters. Indeed some saturation must be observed at longer crystallization times as the crystallinity becomes close to its equilibrium value. No evaluation of the CI at equilibrium was attempted here, but it could be reached by either a short over-cooling or over-stretching of the sample at the end of the acquisition cycle. Crystallization is a two step process consisting of nucleation and growth. Brüning et al. conducted systematic tensile test experiments on NR and they devised a model where growth is the limiting process: part of the chains become taut after stepextension and their ability to coalesce into crystallites is limited by the diffusion processes [31]. The slow-down is caused by the progressive disappearance of free crystallizable segments as they enter crystallites. A relatively complex non-logarithmic kinetic law was derived and the agreement with experimental data is reasonably good as mentioned in their publication. Without discussing the argument that led to discard chain orientation as the 
limiting step, it must be noted that this model is presently in contradiction with the fact that the width of the $(120)+(200)$ equatorial reflections remains constant (this is clearly seen in Figure 3). A progressive lateral growth of crystallites should be reflected by a decrease of the reflection width according to Scherrer's formula [45]. It is our opinion that chain extension is in fact rate determining as it reduces the entropy cost for chain segments entering into the nuclei formation [44]. The growth of oriented crystallites induces a constant relaxation of the local elongation and hence a progressive diminution of the entropy benefit it entails: this could account for the rapid slow-down of the kinetics as crystallization proceeds.

\subsection{Mechanical cycling}

Stress-strain curves have been recorded in a temperature range $0^{\circ} \mathrm{C}-40^{\circ} \mathrm{C}$ with a maximal elongation of around 6; premature breakage is observed at $50^{\circ} \mathrm{C}$. Diffraction patterns were recorded with an indirect illumination CCD X-ray camera at a rhythm of 10s per frame plus $6 \mathrm{~s}$ for the data transfer. In order to obtain a sufficient number of frames per cycle, the strain-rate was fixed at $510^{-3} \mathrm{~s}^{-1}(6 \mathrm{~mm} / \mathrm{min})$. In fact, the logarithmic time-dependence of SIC has for consequence a logarithmic influence of the strain-rate on the stress-strain curves; this has been clearly demonstrated by Candau et al. in the case of NR, as long as the test temperature is well above $T_{g}[44]$.

\subsubsection{Impact of SIC on stress}

Stress-strain curves recorded at the two extremities of the temperature range are displayed in Figure 7-A. Reference stress-strain curves with no SIC could not be obtained due to premature breaking when working at higher temperatures; a Mooney-Rivlin fit is plotted instead as a guide for the eye. The corresponding CI-strain curves are given in Figure 7-B. During stretching we define the elongation $\lambda$ onset for the SIC onset by linear extrapolation to zero of CI (see Figure 7-B); premises of crystallization are detected at ca. $\lambda$ onset -0.4 . Stress hardening is concomitant with $\lambda_{\text {onset }}$ and is evidenced by a departure from the Mooney-Rivlin curve; it is not preceded by stress-softening in contrast to NR [19, 21]. Two cases may be distinguished during recovery depending upon the test temperature: at temperatures lower than around $22^{\circ} \mathrm{C}$, the stress goes to zero at an elongation $\lambda$ Zero before the end of the cycle. The crystalline index cannot be measured below $\lambda$ Zero as the sample starts to bend, but it is expected to remain constant, as depicted in Figure 8-B. At temperatures above $22^{\circ} \mathrm{C}$, the crystalline phase disappears at an elongation $\lambda_{\text {Melting, }}$, below which the mechanical loop nearly closes. 


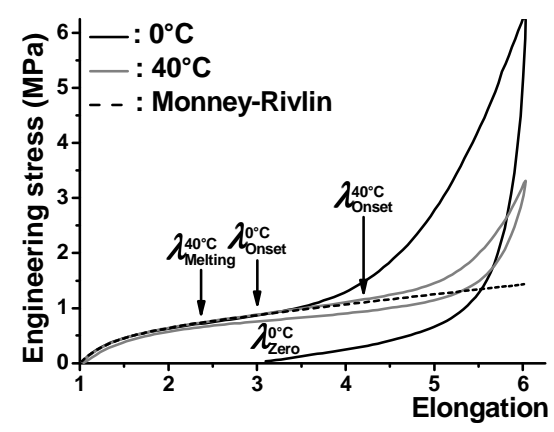

$-\mathrm{A}-$

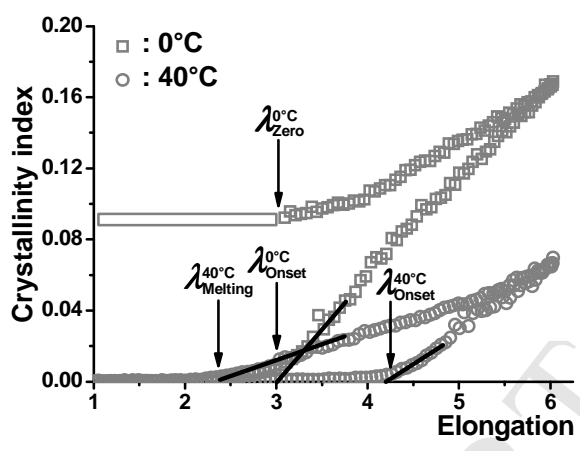

-B-

Figure 7. Left: stress-strain curves at $10^{\circ} \mathrm{C}$ and $40^{\circ} \mathrm{C}$. Right: SIC curves at $10^{\circ} \mathrm{C}$ and $40^{\circ} \mathrm{C}$.

We see in Table 3 that $\lambda_{\text {onset }}$ increases slowly with temperature with values close to those reported for a NR sample with comparable cross-link density [19]. Zhang et al. visually evaluate that an equatorial reflection becomes visible at an elongation of 4 at $20^{\circ} \mathrm{C}$ for a $\mathrm{CR}$ sample with cross-link density $1.910^{4} \mathrm{~mol} / \mathrm{cm}^{3}$ [7]. It is worth comparing $\lambda_{\text {Melting }}$ with values derived from eq.1, keeping in mind that SIC crystallites are grown in static conditions in this latter case. The agreement between both is satisfactory and gives a good estimate for the stability limit of SIC crystallites grown at the different temperatures. Along these lines, one can notice that the estimated temperature of transition between the two regimes $\left(22^{\circ} \mathrm{C}\right)$ is close to the value at $\lambda=1$ derived from eq. $1\left(17.5^{\circ} \mathrm{C}\right)$ : in other terms, the stability limit at zero stress for the crystalline phase grown under SIC conditions is around $20^{\circ} \mathrm{C}$, while complete melting for crystallites grown in the quiescent state only occurs at $37^{\circ} \mathrm{C}$. As crystallographic structural changes cannot be invoked, this significant temperature difference is attributed to different morphologies, most probably a folded conformation in the quiescent state and an extended one for strain-crystallization.

\begin{tabular}{|c|c|c|c|c|c|c|}
\hline Temperature & $\lambda$ Zero & CI at zero stress & $\lambda{ }_{\text {onset }} C R$ & $\lambda_{\text {onset }} N R[15]$ & $\lambda_{\text {Melting }}$ & $\lambda_{\text {Melting }} *$ \\
\hline $0{ }^{\circ} \mathrm{C}$ & 3.1 & 0.094 & 3.0 & --- & --- & --- \\
\hline $10^{\circ} \mathrm{C}$ & 2 & 0.045 & 3.5 & 3.7 & --- & --- \\
\hline $20^{\circ} \mathrm{C}$ & 1.3 & 0.012 & 3.7 & 4.1 & --- & 1.2 \\
\hline $30^{\circ} \mathrm{C}$ & --- & --- & 4.1 & 4.3 & 1.4 & 1.9 \\
\hline $40^{\circ} \mathrm{C}$ & --- & --- & 4.2 & 4.5 & 2.4 & 2.6 \\
\hline
\end{tabular}

Table 3. Relevant elongations associated with mechanical cycling.* eq.1

The amount of mechanical hysteresis versus the area within the CI loop is plotted in Figure 8; it clearly shows that most of the mechanical hysteresis is due to SIC, as previously observed in NR [19]. The small extrapolated value at zero SIC can be attributed to a viscoelastic contribution. 


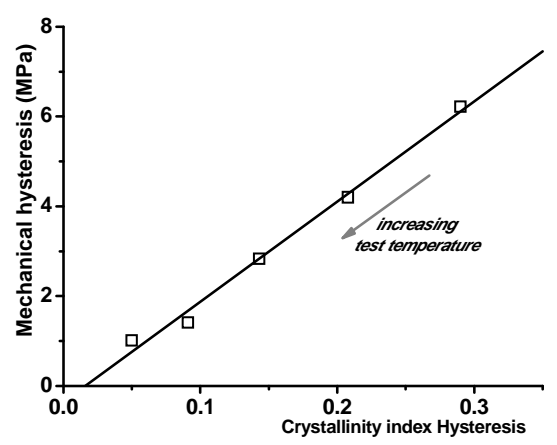

Figure 8. Plot of the mechanical hysteresis observed during cycling versus crystallinity index hysteresis.

\subsubsection{Impact of SIC on the amorphous fraction}

The impact of SIC on strain is less evident than on stress, although it is closely related to the crystallization process itself; It is clearly visible in Figure 9 where the elongationdependence of $\left\langle P_{2}^{R X}\right\rangle$ is plotted for two representative temperatures. During the stretching phase, a clear slow-down of the $\left|\left\langle P_{2}^{R X}\right\rangle\right|$ increase is observed after $\lambda$ onset is reached: this demonstrates the relaxation effect of SIC on strain, that opposes the effect of stretching. During recovery and at the low-temperature regime $\left\langle P_{2}^{R X}\right\rangle$ goes to zero before the zero-stress state $\left(\lambda_{\text {Zero }}\right)$ is reached; we have no explanation for this difference. In the high-temperature regime, the $\left\langle P_{2}^{R X}\right\rangle$-loop closes slightly before the complete melting of the crystalline phase $\left(\lambda_{\text {Melting }}\right)$. It is our opinion that the observed strain-state relaxation has a protective effect that could partly account for the increased resistance to tear imparted by SIC [21].
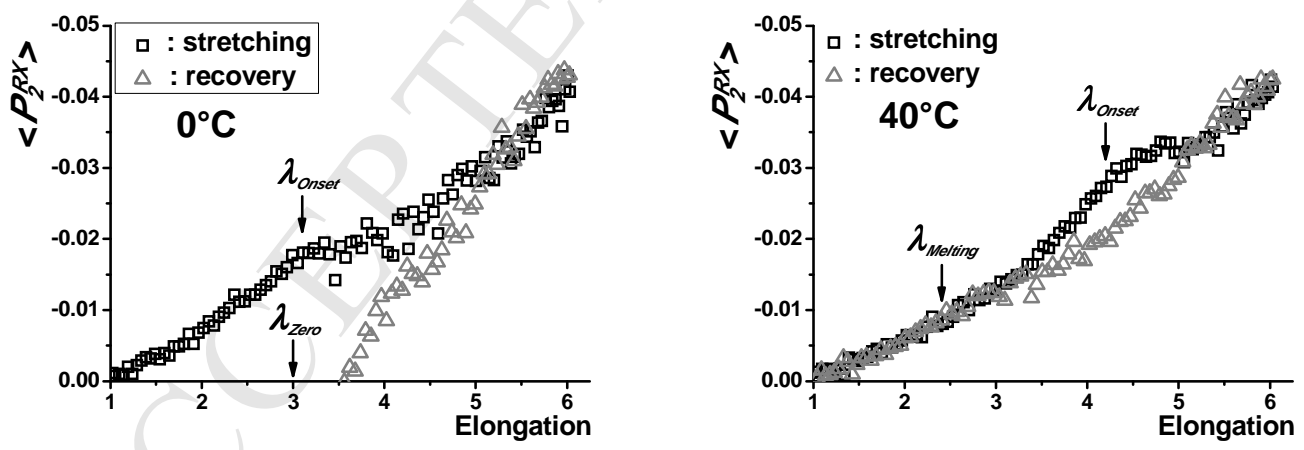

Figure 9. Dependence of the anisotropy parameter $\left\langle P_{2}^{R X}\right\rangle$ upon elongation.

The stress-optical law states that the intrinsic birefringence, or similarly $\left\langle P_{2}\right\rangle$ is proportional to the deviatoric stress in a polymeric liquid (polymer solution or melt). The explanation of this general relationship is that the microscopic origin of stress is chain segment orientation [47]. The Herman coefficient $\left\langle P_{2}\right\rangle$ can be replaced by its X-ray counterpart $\left\langle P_{2}^{R X}\right\rangle$ and we previously used this modified stress-optical law in an attempt to assess the relative contribution of molecular orientation to stress in NR in the absence of crystallization [37]. Figure 10 presents adjustments of the engineering stress before SIC onset by the ratio $K\left\langle P_{2}^{R X}\right\rangle / \lambda$ where $K$ is an adjustment constant and the factor $1 / \lambda$ accounts for the 
decrease of the sample cross-section with elongation. The agreement is satisfactory and implies that the molecular orientation is mainly responsible for the retraction force before SIC onset and that energetic contributions, if present, play a minor role.
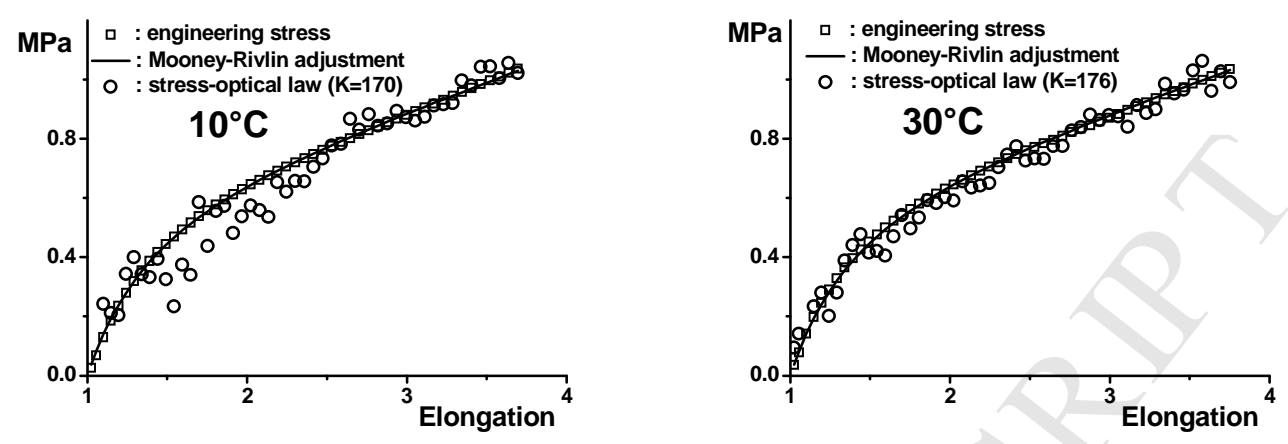

Figure 10.

Adjustments of the engineering stress before SIC onset by the ratio $K\left\langle P_{2}^{R X}\right\rangle / \lambda$

We propose to extend the application of stress-optical law to the region where the sample is partially crystallized by SIC: this is done in Figure 11 for all the investigated temperatures. During stretching and after SIC onset, the contribution of the amorphous fraction to stress starts to level off at ca. 1.2 $\mathrm{MPa}$ at all temperatures; it contributes between $18 \%$ at $0^{\circ} \mathrm{C}$ and $38 \%$ at $40^{\circ} \mathrm{C}$ to the actual stress value at the maximal elongation. This corroborates the attribution made in a preceding study on NR of stress-hardening to crystallite networking [21]. We believe that extensive physical cross-linking by crystallites, or filler effects, cannot be invoked as the origin of stress-hardening: the first effect would result in significantly shorter amorphous chains, and the second in strain-amplification. In both cases a significant increase of the $\left\langle P_{2}^{R X}\right\rangle$ modulus should result. During recovery and at all temperatures, the stress decreases rapidly until an elongation ca. 5.2 is reached where the contribution of the amorphous matrix becomes preponderant. It should be noted that the $\mathrm{CI}$ is still between 0.13 at $0^{\circ} \mathrm{C}$ and 0.04 at $40^{\circ} \mathrm{C}$ at this elongation. This sharp decrease is attributed to the disintegration of the crystallite network [21].
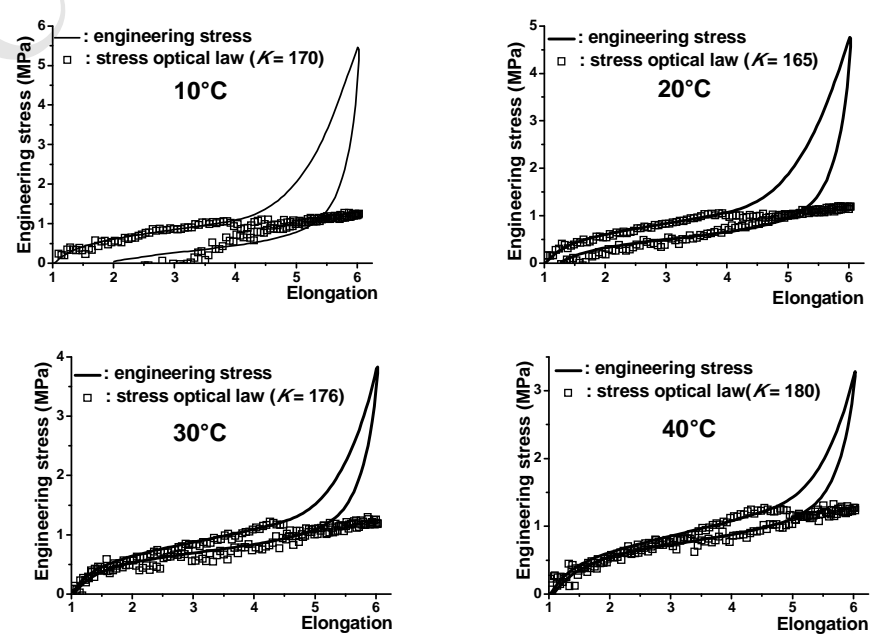
Figure 11. Stress-optical law and engineering stress at different temperatures.

\section{Conclusion}

Combined mechanical and X-ray measurements have helped to clarify the impact of strain-induced crystallization in a polychloroprene rubber. Tensile impact tests for the assessment of the crystallization kinetics could be performed with a satisfying timeresolution, although a simple laboratory X-ray generator was used. An approximately logarithmic time-dependence for the crystalline content is shown; crystallite nucleation is furthermore shown to be a very rapid phenomenon at higher strains. The impact of straininduced crystallization on both stress and amorphous strain during mechanical cycling has subsequently been studied. Strain-induced crystallization is shown to be paradoxically responsible for both stress-hardening and a partial relaxation of the strain-state of the amorphous fraction. Stress-hardening is interpreted in terms of crystallite networking while strain-softening is attributed to strain relaxation similar to the prediction of Flory's theory in static conditions. The rapid decrease in stress observed at the beginning of the recovery part may be due to the disintegration of the crystallite network; the amorphous fraction becomes the only contributor to the stress at still non-negligible crystallinity indices.

A quantitative comparison with natural rubber or other strain-crystallizing polymers is an interesting prospect, but requires samples with similar cross-link densities. However, the lower dependency of the melting temperature upon elongation for the polychloroprene rubber compared to natural rubber is already an indicator of a lesser ability to strain-crystallize. This is reflected by a tendency to break when stretched at temperatures higher than $40^{\circ} \mathrm{C}$. As mentioned above, the role of the important chemical disorder along the polychloroprene chain can be pointed out. It is recalled that a percentage of trans-defects less than $2 \%$ is responsible for a significant degradation of the mechanical properties of synthetic cis-1,4 polyisoprene compared to natural rubber [48].

\section{References}

[1] Le Gac, P. Y., Le Saux, V., Paris, M., \& Marco, Y. (2012). Ageing mechanism and mechanical degradation behaviour of polychloroprene rubber in a marine environment: Comparison of accelerated ageing and long term exposure. Polymer degradation and stability, 97(3), 288-296.

[2] Le Gac, P. Y., Roux, G., Verdu, J., Davies, P., \& Fayolle, B. (2014). Oxidation of unvulcanized, unstabilized polychloroprene: A kinetic study. Polymer Degradation and Stability, 109, 175-183.

[3] Engineering with Rubber, AN. Gent Ed. Hanser Publishers 1992.

[4] Le Gac, P. Y., Broudin, M., Roux, G., Verdu, J., Davies, P., \& Fayolle, B. (2014). Role of strain induced crystallization and oxidative crosslinking in fracture properties of rubbers. Polymer, 55(10), 2535-2542.

[5] Berton, G., Cruanes, C., Lacroix, F., Méo, S., \& Ranganathan, N. (2015). Study of the fatigue behavior of the polychloroprene rubber with stress variation tests. Procedia Engineering, 101, 413-420.

[6] B Huneau, Strain-Induced Crystallization of Natural Rubber: A Review of X-Ray Diffraction Investigations, Rubber Chem. Technol. 84 (2011) 425-452. 
[7] P. Zhang, G. Huang, LL. Qu, YJ. Nie, GS. Weng, JR. Wu, Strain-induced crystallization behavior of polychloroprene rubber, J. Appl. Polym. Sci. 121 (2011) 37-42.

[8] CW. Bunn, Molecular structure and rubber-like elasticity. I. The crystal structure of beta gutta-percha, rubber and polychloroprene, Proc. Roy. Soc. Lond. A 180 (1942) 40-66.

[9] J. Che, C. Burger, S. Toki, LX. Rong, BS. Hsiao, Crystal and Crystallites Structure of Natural Rubber and Synthetic cis-1,4-Polyisoprene by a New Two Dimensional Wide Angle X-Ray Diffraction Simulation Method. I. Strain-Induced Crystallization, Macromolecules 46 (2013) 4520-4528.

[10] V. Grmela, L. Megarskaya, The kinetics of crystallization of polychloroprene rubber, Vysokomol. Soyed A10 (1968) 1032-1037.

[11] AN. Gent, S. Kawahara, J. Zhao, Crystallization and strength of natural rubber and synthetic cis-1, 4polyisoprene, Rubber Chem. Technol. 71 (1998) 668-678.

[12] D. Luch, GSY. Yeh, Morphology of strain-induced crystallization of natural rubber. I. Electron microscopy on uncrosslinked thin film, J.Appl.Phys. 43 (1972) 4326-4338.

[13] H. Yoshimoto, S.Sagae, M.Matsuo, S.Uemara, Y. Ishida, Crystalline texture of polychloroprene as revealed in ultrathin sections, Kolloid Z. Z. Polym. 236 (1970) 116-118.

[14] J. Che, C. Burger, S. Toki, LX. Rong, BS. Hsiao, Crystal and Crystallites Structure of Natural Rubber and Peroxide-Vulcanized Natural Rubber by a New Two Dimensional Wide Angle X-Ray Diffraction Simulation Method. II. Strain-Induced Crystallization versus Temperature-Induced Crystallization, Macromolecules 46 (2013) 9712-9721.

[15] AN. Gent, Crystallization in stretched polymer networks I. trans-polychloroprene, Rubber Chem. Technol. 40 (1967) 1071-1083.

[16] WR. Krigbaum, JV. Dawkins, HH. Via, Crystallite orientation in stretched polycloroprene networks.II., J. Polym. Sci. A2 7 (1969) 257-270.

[17] GSY. Yeh, Strain-induced crystallization II. Subsequent fibrillary-to-lamellar transformation, Polym. Eng. Sci. 16 (1976) 145-151.

[18] YJ. Nie, HH. Gao, WB. Hu, Variable trends of chain-folding in separate stages of strain-induced crystallization of bulk polymers, Polymer 55 (2014) 1267-1272.

[19] S. Trabelsi, P-A. Albouy, J. Rault, Crystallization and melting process in vulcanized stretched natural rubber, Macromolecules 36 (2003) 7624-7639.

[20] PJ. Flory, Thermodynamics of Crystallization in High Polymers. I Crystallization Induced by Stretching, J. Chem. Phys. 15 (1947) 397-408.

[21] P-A. Albouy, A. Vieyres, R. Pérez-Aparicio, O. Sanséau, P. Sotta, The impact of strain-induced crystallization on strain during mechanical cycling of cross-linked natural rubber, Polymer 55 (2014) 40224031.

[22] RR. Garett, CA. Hargreaves, DN. Robinson, The Structurally Regular trans-1,4-Poly(chloroprene), J. Macromol. Sci., Chem. 48 (1970) 1679-1703.

[23] JE. Mark, The Constants $2 C_{1}$ and $2 C_{2}$ in Phenomenological Elasticity Theory and Their Dependence on Experimental Variables. Rubber Chem. Technol., 48 (1975), 495-512 
[24] P-A. Albouy, G. Guillier, D. Petermann, A. Vieyres, O. Sanséau, P. Sotta, A stroboscopic X-ray apparatus for the study of kinetics of strain-induced crystallization in natural rubber, Polymer 53 (2012) 3313-3324.

[25] H. Kawai, Dynamic X-ray diffraction technique for measuring rheo-optical properties of crystalline polymeric materials, Rheol. Acta 14 (1975) 27-47.

[26] N. Candau, L. Chazeau, J-M. Chenal, C. Gauthier, J. Ferreira, E. Munch, D. Thiaudière, Strain induced crystallization and melting of natural rubber during mechanical cycles, PCCP 17 (2015) 15331-15338.

[27] C. Ponchut, F. Zontone, H. Graafsma, Experimental comparison of pixel detector arrays and CCD-based systems for X-ray area detection on synchrotron beamlines, IEEE T. Nucl. Sci. 52 (2005) 1760-1765.

[28] MJH. Koch, J. Bordas, E. Schöla, HC. Broecker, Kinetic study of the crystallization of stretched polyisobutylene using synchrotron radiation, Polym. Bull. 1 (1979) 709-714.

[29] M. Tosaka, K. Senoo, K. Sato, M. Noda, N. Ohta, Detection of fast and slow crystallization processes in instantaneously-strained samples of cis-1.4-polyisoprene, Polymer 53 (2012) 864-872.

[30] K. Brüning, K. Schneider, SV. Roth, G. Heinrich, Kinetics of Strain-Induced Crystallization in Natural Rubber Studied by WAXD: Dynamic and Impact Tensile Experiments, Macromolecules 45 (2012) 7914-7919.

[31] K. Brüning, K. Schneider, SV. Roth, G. Heinrich, Kinetics of strain-induced crystallization in natural rubber: A diffusion-controlled rate law, Polymer 72 (2015) 52-58.

[32] JH. Wendorff, The structure of Amorphous Polymers, Polymer 23 (1982) 543-557.

[33] WR. Krigbaum, RJ. Roe, Diffraction study of Crystallite orientation in a stretched polychloroprene vulcanizate, J. Polym. Sci. A2 (1964) 4391-4414.

[34] M Kakudo and N Kasai, X-Ray Diffraction by Polymers, Kodansha Ltd and Elsevier Publishing Company, 1972 .

[35] HP. Klug, LE. Alexander, X-Ray Diffraction Procedures For Polycrystalline and Amorphous Material, Wiley, New-York, 1954.

[36] HJ. Wang, J. Zhou, Numerical conversion between the Pearson VII and pseudo-Voigt functions, J. Appl. Cryst. 38 (2005) 830-832.

[37] M. Zaghdoudi, P-A. Albouy, Z. Tourki, A. Vieyres, P. Sotta, Relation between stress and segmental orientation during mechanical cycling of natural-based compound, J. Pol. Phys. 53 (2015) 943-950.

[38] GR. Mitchell, AH. Windle, Measurement of molecular orientation in thermotropic liquid crystalline polymers, Polymer 24 (1983) 1513-1520.

[39] GR. Mitchell, A wide-angle X-ray study of the development of molecular orientation in crosslinked natural rubber, Polymer 25 (1984) 1562-1572.

[40] B. Amran, L. Bokobza, J-P. Queslel, L. Monnerie, Fourier-transform infra-red dichroism study of molecular orientation in synthetic high cis-1,4-polyisoprene and in natural rubber, Polymer 27 (1986) 877-882.

[41] M. Tosaka, S. Kohjiya, Y. Ikeda, S. Toki, BS. Hsiao, Molecular orientation and stress relaxation during strain-induced crystallization of vulcanized natural rubber, Polym. J. 42 (2010) 474-481. 


\section{ACCEPTED MANUSCRIPT}

[42] S. Poompradub, M. Tosaka, S. Kojiya, Y. Ikeda, S. Toki, I. Sics, B. Hsiao, Mechanism of strain-induced crystallization in filled and unfilled natural rubber vulcanizates, J. Appl. Phys. 97 (2005) 103529.

[43] K. Loos, M. Johlitz, A. Lion, L. Palgen, J. Calipel "New ideas to represent strain induced crystallization in elastomers" in "Constitutive Models for Rubber X, Lion and Johlitz Eds., Taylor \& Francis Group (2017).

[44] PA. Albouy and P. Sotta in Polymer crystallization II From Chain Microstructure to Processing, F. Auriemma, G.C. Alfonso, C.D. Rosa Eds., Springer (2017).

[45] JI. Langford, AJC. Wilson, Scherrer after Sixty Year: A Survey and Some New Results in the Determination of Crystallite Size, J. Appl. Cryst. 11 (1978) 102-113.

[46] N. Candau, R. Laghmach, L. Chazeau, J-M. Chenal, C. Gauthier, T. Biben, E. Munch, Influence of strain rate and temperature on the onset of strain induced crystallization in natural rubber, Eur. Polym. J. 64 (2015) $244-252$.

[47] M. Doi, S.F. Edwards, The Theory of Polymer Dynamics; Clarendon Press: Oxford, 1995.

[48] S. Trabelsi, P-A. Albouy, J. Rault, Strain-induced crystallization properties of natural and synthetic cispolyisoprene, Rubber Chem. Technol. 77 (2004) 303-316. 